\title{
Motivational telephone intervention to risk gamblers by a state-owned gambling operator in Sweden
}

Anders Håkansson ( $\square$ anders_c.hakansson@med.lu.se )

Lund University

Katja Franklin

AB Svenska Spel

Maria Dahlmström

AB Svenska Spel

Axel Lyckberg

AB Svenska Spel

\section{Research Article}

Keywords:

Posted Date: March 7th, 2022

DOI: https://doi.org/10.21203/rs.3.rs-1289602/v1

License: (c) (1) This work is licensed under a Creative Commons Attribution 4.0 International License. Read Full License 


\section{Abstract}

Background: Few studies have tested the effect of a motivational telephone intervention from a gambling operator to clients with high-risk gambling practices. This study aimed to study subsequent gambling abstinence, limit setting and self-exclusions post-intervention, compared to controls.

Methods: The study assessed a motivational, personalized telephone intervention by the state-owned Swedish gambling operator AB Svenska Spel. Clients successfully reached with the telephone intervention $(n=1,420)$ were compared to clients who could not be reached $(n=1,504)$. Gambling practices during eight weeks pre-intervention were assessed, and outcome measures were gambling abstinence, limit setting and self-exclusion four weeks post-intervention.

Results: The telephone intervention was associated with increased gambling abstinence weeks 2-4 postintervention ( 24 vs 20 percent, $p=0.02$ ), limit settings ( 10 vs 5 percent, $p<0.001)$ and self-exclusions ( 11 vs 8 percent, $p<0.01$ ), but not significantly associated with full week 1-4 abstinence (18 vs 15 percent, $p=0.07)$. In unadjusted analyses of sub-groups, significant associations of the intervention with full gambling abstinence were seen in online casino/bingo gamblers (19 vs 14 percent, $p<0.01$ ). In logistic regression, controlling for different gambling types, effects of the intervention were seen in the highest sub-segment with respect to gambling losses.

Conclusions: A personalized motivational telephone intervention to high-risk gamblers, delivered by a gambling operator, is promising. Effects are likely more pronounced in players of online chance-based games, and in gamblers with high pre-intervention losses.

\section{Background}

Gambling disorder is an addictive disorder characterized by extensive gambling habits, typically with a high level of loss of control, and continued gambling despite severe financial and other consequences. Gambling disorder is often associated with severe mental health consequences [1, 2].

In recent years, gambling operators' responsibility for the detection of emerging gambling problems has been highlighted in policy work and research [3-6]. Responsible gambling tools used by gambling operators may include monitoring of gambling patterns with the aim to detect problem gambling, and may also involve the provision of harm-reducing tools such as self-exclusion, or voluntary limit setting by gamblers aiming to cut down on their gambling [7]. In particular, online-based gambling services technically may allow for the detection of emerging gambling problems in close temporal association with the gambling sessions, and the detection of major financial losses may constitute a window of opportunity for such interventions to take place.

In 2019, the state-owned gambling operator of Sweden, AB Svenska Spel, initiated a responsible gambling intervention. This intervention aims to identify gamblers with high-risk gambling patterns, and to carry out personalized telephone calls to these gamblers, with a motivational aim of helping gamblers 
reflect upon their gambling patterns, motivate them for self-exclusion or limit-setting measures, or to seek treatment or other support services. The telephone intervention is carried out by trained officers of the gambling operator. The overall project of motivational telephone calls by AB Svenska Spel has been described in the protocol paper preceding the present results paper [8].

A motivational interviewing approach to problem gamblers has been demonstrated to be efficacious in order to reduce gambling [9]. The delivery of a telephone intervention from a gambling operator, directly addressing gamblers with high-level gambling practices, has been shown to be promising in the limited extent of research hitherto carried out. In an intervention from the state-owned Norwegian gambling operator, direct telephone contact proved to be more efficacious on subsequent gambling practices than a postal letter contact $[10,11]$. However, studies on these telephone interventions are few, and there is need for increased knowledge about their potential effects in high-risk gambling. Also, in addition to the potential for an intervention to clients with detected high-risk behaviors, it has not hitherto been demonstrated what value such responsible gambling interventions have with gamblers who voluntarily take a self-test, i.e. where a first step in the motivational process already can be suspected to have occurred.

Therefore, the present study aimed to analyze, in a controlled study design, 1) whether a motivational, personal telephone intervention to high-risk gamblers was associated with gambling abstinence and selflimiting practices, in comparison to controls who were intended for the same intervention but who could not be reached, and 2) which factors may be associated with gambling abstinence and self-limiting practices in the assessed study group, and 3 ) the time frame from the intervention to self-exclusion postintervention.

\section{Methods}

The study protocol of the present study has been published [8]. The data presented here refer to the quantitative controlled effectiveness study. Remaining study parts, including data describing users' satisfaction with the intervention and post-intervention self-reported gambling data, are to be reported elsewhere.

\section{Setting}

The Swedish gambling market is a license-based market since January 2019, with a high number of registered gambling operators in competitive gambling markets such as sports betting, online casino and bingo gambling, lotteries and online poker. As exceptions to this, land-based casinos (at three sites in the country) and land-based electronic gambling machines are provided as part of a state-owned monopoly. One sub-division of the state-owned gambling operator AB Svenska Spel provides the latter monopoly services, whereas one sub-division of the company provides chance-based lottery games (Svenska Spel Tur, translated into 'luck') on a competitive market, and a third sub-division provides sports- and chancebased games (Svenska Spel Sport \& Casino) on a competitive market. The present study refers to the 
latter, i.e. the sub-division providing different types of sports betting (where bets can be made either online or in specific gambling stores, online casino, online bingo gambling and online poker).

Problem gambling in Sweden is reported in about 1.5 percent of the general population, with some fluctuations over the years [12]. Treatment seeking for gambling disorder is in a large majority of cases based on problematic online gambling, in contrast to more traditional land-based gambling types [13]. Gambling advertisements seen in Swedish television are predominantly promoting online casino gambling and online sports betting [14]. Treatment is available from social services of municipalities and from a limited number of health-care institutions [15].

\section{Study participants}

Participants were included if they fulfilled the project's criteria of reaching above a certain level of monetary loss, or because they were taking the self-test GamTest and coded as 'red' (indicating a high risk of having a gambling problem) according to that test [16]. The present study includes motivational telephone calls from the month of September, 2019, through April, 2020. The distribution of telephone interventions/attempts registered per month are displayed in Table 1.

Data were available from a total of 3,562 individuals (who were included only once, or, who were included on their first attempt, provided the second attempt was at least four weeks beyond the first occasion). Intervention (successful call) was registered in 1,420 individuals, whereas the control condition (not reached for three contact attempts) was registered for 1,504 individuals. Further, 241 unique individuals were reached but did not want to talk, 30 responded but were unable to carry out the call, 13 were not contacted because of a recent self-exclusion activated, and in 334 individuals, adequate contact details were unavailable. In 20 individuals, no outcome of the telephone was registered. Thus, intervention $(n=1,420)$ and control subjects $(n=1,504)$ were included in the study (a total of 2,924 individuals).

\section{Study procedure}

The intervention was defined as a motivational intervention call successfully carried out and completed, and this was compared to the control condition, which was defined to occur in individuals who were not reached despite three attempts. Occasions when the gambler was reached but did not wish to talk, or when the talk could not be carried out for other (such as technical) reasons, were excluded from further study. The nature and content of the intervention calls are described in Figure 1. As described there, the nature of the telephone conversation is primarily motivational and non-judgmental, and its content depends on the degree of motivation, change talk and intentions of the client. Thus, the conversion may assist subjects in self-excluding or gambling-reducing measures whenever the clients wishes to, and may facilitate gambling abstinence for clients who are perceived to with for that, but also may have a content earlier in a motivational process for clients with obvious intention to reduce gambling. 
As part of the analytical strategy of the present study, the original intention was to match (in a 1:1 ratio) individuals exposed to the intervention to control individuals [8]. In the present analyses, we chose not to proceed with the matching procedure, in order to limit the number of individual data lost, and in order to be able to conduct regression analyses controlling for a number of relevant variables, as it was increasingly evident that co-factors such as gambling types may need to be taken into account in regression analyses. Here, in order to be able to study the role of gambling extent for the outcomes assessed in the study, we included the level of past-28-day gambling losses as one of the predictors and one of the variables controlled for. In addition, for the main outcome in the study (gambling abstinence after the motivational call or attempts for the motivational call), this analysis was carried out separately for five different sub-segments following their level of 28-day losses. Thus, the overall strategy instead was a logistic regression analysis taking gambling losses into account, rather than a case-control procedure matching individuals based on losses.

Also, in addition to the strategy described in the protocol paper [8], we chose to assess both full gambling abstinence after the day of the intervention (or the day of the final attempt for the control group), and gambling abstinence for only the three last study weeks, i.e. week 2-4 after the intervention or control contact attempt. The reason for this additional analysis is that for any gambling, and for gambling involving the planning of bets during a few days prior to the event (such as sports betting for a specific sports event), it may be counter-intuitive that an intervention would immediately lead to gambling abstinence already on the day of the intervention and on the following few days. For this reason, in this additional analysis, post hoc we consider the first week to be a potential period for a motivational procedure to start.

The study period comprised the eight weeks preceding the telephone call, and the four weeks subsequent to the call. Time point zero, T0, was defined as the date of the call, or the day of the third attempt in the control group. Thus, T0 was the first day of the first week, of the four-week follow-up period (postintervention weeks 1-4). As described below, the main analyses of the potential effect of the intervention were logistic regression analyses, in which intervention (vs control) was included as one of the potential independent variables, along with demographic data (age, gender), whether a self-test was available (as this was one of the inclusion criteria used for the overall intervention project), the level of past 28-day losses on the Sports \& Casino gambling services), and whether or not the individual had any gambling occasion registered for the pre-T0 period of the study for each of the gambling types included (pool sports betting, other sports betting, online poker, online slots gambling, other online casino gambling, or online bingo gambling). The distribution of pre-T0 gambling on different gambling types is shown in Table 2.

All data were derived from the client registers of AB Svenska Spel, and delivered to the first author in a fully de-identified format. The study was approved by the Swedish Ethics Authority (file number 2020/03281), and it was pre-registered on clinicaltrials.gov with the clinical trials identifier number NCT04646421 (registered 30/11/2020). Based on the ethics decision, no informed consent was obtained from study participants. 


\section{Variables}

Extent and frequency of gambling within Svenska Spel Sports \& Casino

- Gambling frequency (days per week, 0-7 days, here calculated as the mean of the eight weeks prior to T0, and for the four weeks post-T0, respectively).

- Level of losses during the past 28 days prior to T0 (losses on Svenska Spel Sports \& Casino gambling services, in SEK (approximately nine SEK corresponding to one USD), categorized into 2,000 SEK segments ranging from $0-1,999,2,000-3,999$, etc, through a maximum level of 40,000 and higher)

Gambling types used, defined as the occurrence of any gambling registration of a gambling type during any of the eight weeks preceding T0:

- Pool sports betting games

- Sports betting, other (online or in licensed land-based gambling stores)

- Poker games online

- Casino slots gambling online

- Live online casino gambling

- Online casino, other (other chance-based casino games, including casino table games, video poker, and virtual sports betting)

Age (in years)

Gender (male, female, missing), here analyzed as female gender vs male gender

Red self-test available

Content of the telephone intervention:

- Advice about limit setting was given (yes/no)

- Advice for self-exclusion was given (yes/no)

- Information given about gambling history (yes/no)

Outcome measures post-T0:

- Gambling abstinence post-T0 (no registration of gambling on Svenska Spel Sports \& Casino post-T0, week 1-4)

- Gambling abstinence week 2-4 post-T0 (no registration of gambling on Svenska Spel Sports \& Casino during the weeks 2-4 post-T0, i.e. regardless of gambling data during the first week post-T0)

- Self-exclusion, any time post-T0 during week 1-4, as well as time from T0 to self-exclusion (days). Aggregated measure of any choice for self-exclusion (including self-exclusion for one day, one week, 
or one, three, six, 12 or 36 months, for one or all gambling types).

- Limit setting, any time post-T0 during week 1-4 (any limit which is either the first limit or a lowered limit compared to a previous one). Aggregated measure of any limit setting carried out (including loss limits, and deposit limits, per day, week or month, respectively).

\section{Statistical methods}

Group comparisons regarding categorical variables, such as comparing categorial descriptive or outcome data between the intervention and control groups, were made using the chi-square test. Corresponding group comparisons for continuous variables (age) were made using Student's t test. Group comparisons of continuous variables in smaller subsets of individuals (such as days to self-exclusion) were made using the Mann-Whitney U test and compare medians instead of mean values. Descriptive data were reported as number and percentages, and for continuous variables, as mean values and median values, with distribution measures of standard deviation (SD) and inter-quartile ranges (IQR), respectively.

Non-stepwise logistic regression analyses were carried out for the following outcome variables: gambling abstinence week 1-4, gambling abstinence week 2-4, self-exclusion, and limit settings. In these analyses, we included, as independent variables, intervention vs control, age, gender, presence of a self-test, level of past-28-day gambling losses (categorized in 2,000 SEK sub-segments included as a continuous variable), and the occurrence (dichotomous, yes/no) of pre-T0 gambling for each of the separate gambling types. For the outcome measure of full gambling abstinence (week 1-4), separate logistic regression analyses were carried out for each of sub-segments of gambling losses $(<10,000,10,000-19,999,20,000-29,999$, 30,000-39,999, and 40,000+ SEK), and for each gender and for individuals with any chance-based online gambling (casino gambling types and bingo) and with any type of sports betting (pool or other sports betting).

Prior to the logistic regression analyses, in order to reduce the risk of inter-collinearity, a correlation matrix was run, including all variables used as independent variables. This correlation matrix' highest values of correlations were seen for the correlation between red self-test and level of losses $(r=0.43)$, between pool sports betting and other sports betting $(r=0.43)$, and between live casino and other casino gambling $(r=0.33)$. Other correlations were at 0.23 (live casino gambling and age) and below. None of the correlation levels demonstrated were judged to disqualify any of the variables from the regression analyses.

Due to the change in the gambling market during the spring months of 2020, due to the COVID-19 pandemic [17,18], a sensitivity analysis was carried out, excluding subjects included during the month of April, 2020. In this sensitivity study, the main logistic regression analyses (gambling abstinence, abstinence post-T0 week 2-4, self-exclusion, and limit setting) were carried out in all individuals with a T0 date from September, 2019, through March, 2020, i.e. excluding subjects with a T0 date in April, 2020. 
Prior to that, group comparisons were made between individuals included in April, vs all others, in order to highlight potential differences in key variables.

All statistical calculations were made in the SPSS software [19].

\section{Results}

A total of 2,924 individuals, who were either successfully reached (intervention) or who were called but not reached (control) were included in the study. Among them, 241 (eight percent) were women, 2,682 (92 percent) were men, and gender was missing for one individual. Mean age (missing in nine individuals) was 38.3 years (SD 13.9), with a median age of 36 years (range 18-79, IQR 25-49).

The mean number of weekly gambling days was 3.3 prior to TO (SD 2.1, median 3.0, IQR 1.5-5.1), and 2.7 after T0 (SD 2.25, median 2.25, IQR 0.5-4.5). The median level of past-28-day gambling losses (available in 2,686 individuals) was 14,121 SEK (IQR 5,500-31,119 SEK). Self-tests were available in 506 individuals (17 percent).

Group differences in baseline characteristics between intervention and control subjects are displayed in Table 3.

\section{Role of content in the telephone intervention}

Among telephone interventions carried out, if advice about limit setting was delivered, the chance of a gambling limit set post-T0 was significantly higher ( 13 vs $5 \%, p<0.001)$, whereas the association with gambling abstinence (13 vs 23 percent, $p<0.001$ ) and with week $2-4$ abstinence (18 vs 30 percent, $p<0.001)$ were instead negative. If advice for self-exclusion was given, the chance of a self-exclusion (16 vs 6 percent, $p<0.001)$ and the chance of gambling abstinence (22 vs 14 percent, $p<0.001$ ) or week $2-4$ abstinence ( 27 vs 21 percent, $p=0.01$ ) were significantly higher. If advice about gambling history information was delivered, gambling abstinence was lower (13 vs 28 percent, $p<0.001$ ), as well as week 24 abstinence ( 20 vs 32 percent, $p<0.001$ ), whereas no association was seen with self-exclusion ( 10 vs 13 percent, $\mathrm{p}=0.13)$.

\section{Self-exclusion and limit setting}

Self-exclusion post-intervention ( 9 percent, $n=268)$ was significantly more common $(p<0.01)$ in the intervention group (11 percent, $n=154$ ) than in the control group (8 percent, $n=114$ ). Limit setting postintervention was seen in seven percent $(n=213)$, more commonly $(p<0.001)$ in the intervention group (10 percent, $n=137$ ) than in the control group ( 5 percent, $n=76$ ).

Self-exclusion was, in logistic regression (Table 7), significantly associated with older age $(p<0.001)$, and positively associated with live casino gambling ( $<<0.001)$, slots casino gambling $(p<0.001)$, and with the 
intervention ( $p<0.01)$. Limit setting, in logistic regression (Table 8), was significantly and negatively associated with pool sports betting $(p<0.001)$, and positively associated with online casino gambling $(p=0.04)$ and with the intervention $(p<0.001)$.

\section{Time to self-exclusion in the intervention group}

For respondents who self-excluded following the intervention call, $45(n=69)$ of all self-exclusions $(n=154)$ were made during the day of the call, and the median time from the intervention call to self-exclusion was one day (IQR 0-13 days, mean 6.6 days, SD 8.5). A longer time to self-exclusion post-intervention (MannWhitney $U$ test due to the low number of data) was associated with female gender (19.5 vs 1 days, $p=0.01$ ), but not with casino/bingo gambling ( 3 vs 0 days, $p=0.12$ ), whereas it was not associated with sports betting ( 1 vs 1 days, $p=0.65$ ).

\section{Gambling abstinence post-T0}

Rates of gambling abstinence, and week 2-4 abstinence, are displayed in Table 4, for all subjects and for specific sub-groups.

Gambling abstinence post-T0 was detected in 16 percent $(n=480)$. This was not significantly higher in the intervention group (18 percent, $n=251$ ) than in the control group ( 15 percent, $n=229, p=0.07$ ). Gambling abstinence post-T0 during week 2-4 post-intervention was seen in 22 percent $(n=635)$, and significantly more in the intervention group ( 24 percent, $n=334$ ) than in the control group ( 20 percent, $n=301, p=0.02$ ).

Post-T0 gambling abstinence was, in logistic regression (Table 5), significantly associated with younger age $(p<0.001)$ and with the presence of a self-test $(p<0.001)$, and significantly and negatively associated with pre-T0 pool sports betting $(p<0.001)$, other sports betting $(p<0.001)$, online poker gambling $(p<0.001)$, and online slots gambling $(p<0.01)$. Here, the intervention was not significantly associated with gambling abstinence $(p=0.08)$.

Post-T0 gambling abstinence during weeks 2-4 was, in logistic regression (Table 6), significantly associated with younger age $(p<0.001)$, and negatively associated with pool sports betting $(p<0.001)$, other sports betting $(p<0.001)$, online poker gambling $(p<0.001)$ and slots gambling $(p<0.001)$, and also associated with the presence of a self-test $(p<0.01)$, and positively and significantly associated with the intervention $(p=0.01)$.

\section{Gambling abstinence post-T0 in sub-segments with different levels of gambling losses}

In clients with less than 10,000 SEK of 28-day losses pre-T0 ( $n=1,075$, among whom 1,072 with full data in the regression analysis), gambling abstinence was significantly associated with the presence of a self- 
test $(p<0.001)$, and negatively associated with pool sports betting $(p<0.001)$ and other sports betting $(p<0.001)$, online poker $(p=0.02)$, and online slots gambling $(p<0.001)$. In the sub-segment with 28 -losses of 10,000-19,999 SEK ( $n=523$, among whom 520 with full data), gambling abstinence was negatively associated with pool sports $(p<0.001)$ and other sports betting $(p<0.01)$ and with online poker gambling ( $p<0.001)$. In the 20,000-29,999 SEK sub-segment ( $n=364$ with full data), gambling abstinence was associated with younger age $(p=0.03)$, pool sports $(p=0.04)$ and other sports betting $(p<0.001)$. In the 30,000-39,999 SEK sub-segment ( $n=333$ with full data), these associations were seen for younger age $(p<0.01)$ and pool sports betting $(p<0.01)$. Thus, in all sub-segments described above, no significant association was seen with the study intervention. In the sub-segment with losses of 40,000 SEK or more $(n=391$, among whom 390 with full data), gambling abstinence was significantly associated with the intervention ( $p=0.03$, OR 1.93 [1.07-3.45]), pool sports betting $(p=0.01)$, and poker gambling $(p=0.03)$. Other variables in these sub-segments had no significant association with gambling abstinence, and, thus, the intervention vs control was significantly associated with gambling abstinence only in the segment above 40,000 SEK of 28 -day losses.

\section{Different gambling types}

Among gamblers with online casino/bingo gambling prior to $\mathrm{T} 0$, the intervention was positively and significantly associated with post-T0 gambling abstinence (OR 1.44 [1.07-1.93], $p=0.01$ ), week 2-4 gambling abstinence (OR 1.31 [1.01-1.71], $p=0.04$ ), self-exclusion (OR 1.47 [1.09-1.98], $p=0.01$ ), and limit setting (OR 2.05 [1.39-3.01], $p<0.001$ ), when controlling for the other variables included in the logistic regression analyses. In the corresponding logistic regressions, all sports bettors (whether or not they also gambled on chance-based gambling), the intervention was positively and significantly associated with post-T0 self-exclusion (OR 1.77 [1.29-2.43], p<0.001) and with limit setting (OR 2.49 [1.75-3.53], $p<0.001)$, and with week 2-4 gambling abstinence (OR 1.28 [1.01-1.61], $\mathrm{p}=0.04)$, but not associated with gambling abstinence (OR 1.14 [0.87-1.48], $p=0.34$ ).

\section{Sensitivity analysis with respect to potential effects COVID- 19-related effects}

Individuals included with a T0 date in April ( $\mathrm{n}=391)$, were, compared to all other included subjects $(n=2,533)$, less likely to have a pre-T0 gambling on pool sports (58 vs 75 percent, $p<0.001)$, other sports betting (48 vs 66 percent, $p<0.001$ ), and more likely to have a pre-T0 online poker gambling (28 vs 20 percent, $p<0.001$ ), online slots gambling ( 51 vs 42 percent, $p<0.001$ ), live casino gambling (31 vs 25 percent, $p<0.01$ ), but no difference with respect to online bingo ( 9 vs 7 percent, $p=0.13$ ) and other casino gambling (11 vs 10 percent, $p=0.50$ ). Altogether, subjects included in April were significantly more likely to have pre-T0 gambling on any casino/bingo gambling ( 66 vs 55 percent, $p<0.001$ ) or any sports betting (64 vs 83 percent, $p<0.001$ ). Also, subjects included in April were significantly more likely to be female (12 vs 8 percent, $p=0.01$ ), less likely to be reached by the intervention call ( 44 vs 49 percent, $p=0.04$ ), more 
likely to have post-T0 gambling abstinence (30 vs 14 percent, $p<0.001$ ), week $2-4$ gambling abstinence (35 vs 20 percent, $p<0.001$ ), self-exclusion ( 19 vs 8 percent, $p<0.001$ ), but less likely to have a post-0 limit setting ( 3 vs 8 percent, $p<0.001$ ), whereas no difference was seen with respect to the presence of a selftest ( 20 vs 17 percent, $p=0.14$ ).

In logistic regression analyses, when excluding subjects included in April (and controlling for the same variables as in the main analyses), the intervention (vs control) was not significantly associated with gambling abstinence (OR 1.23 [0.96-1.58], $p=0.11$ ), but significantly associated with week 2-4 gambling abstinence (OR 1.29 [1.04-1.61], $p=0.02$ ), with self-exclusion (OR 2.05 [1.48-2.84], $p<0.001$ ), and with limit setting (OR 2.39 [1.72-3.31], $p<0.001)$.

\section{Discussion}

The present study is one of few assessing an intervention where a gambling operator, as a part of its responsible gambling policy, carries out a personal, telephone-based, motivational interventions targeting individuals with hazardous gambling practices. While the unadjusted group comparison between subjects reached by the intervention and control subjects did not demonstrate a significant effect on complete gambling abstinence following the motivational call, the measure of gambling abstinence 2-4 weeks after the intervention demonstrated a significant effect of the intervention. In the adjusted analyses, controlling for demographic data and for different gambling types, an independent association with gambling abstinence was seen in the highest sub-segment of gambling losses, but not in the lower ranges of gambling losses.

When controlling for gambling types, age, gender, self-test, level of losses, the intervention was significantly associated with limit setting post-intervention, with post-intervention self-exclusions, and with week 2-4 non-gambling whereas it was not associated with non-gambling post-intervention.

Altogether, in different aspects, the motivational telephone intervention described here demonstrated promising effects on key components of gambling practices. The aim of the motivational intervention is not primarily to obtain gambling abstinence unless this is perceived to be the client's desire to obtain, but more typically introduces and reinforces a motivational process which may contribute to reduced gambling habits. Despite this, and although gambling abstinence is not necessarily an intention of the call, an effect on gambling abstinence could be seen when analyzing the weeks $2-4$ after the intervention, while an effect could not be demonstrated with respect to full gambling abstinence immediately after the intervention. Here, it should be borne in mind that the intervention is not a selfselected intervention sought out by the gambler, but instead an intervention happening without any preparation from the user's side, and where it must be seen as unlikely that a substantial effect would occur at once. Still, having said that, one important conclusion from the present study is that the associations with gambling abstinence were markedly more pronounced in some groups; significant and clearly larger effects were seen in the highest sub-segment of recent losses, in men, and in gamblers with any online casino/bingo gambling. 
The effects of the intervention appeared to be more robust for the harm-reducing gambling tools, selfexclusion and limit setting, than for the actual outcome of gambling abstinence. Here, it can be argued that the personal motivational contact may more clearly facilitate the decision to self-exclude or limit one's gambling, and that it was clearly less likely that these events would occur during this specific follow-up period in subjects who were not reached by the intervention. This is further strengthened by the fact that when self-exclusion or limit-setting advice was noted as a topic addressed during the conversation, this further increased the likelihood that such measures were taken, either in direct association with the call, or later.

Self-exclusion advice in the telephone conversation was associated with actual self-exclusion, and, logically, also with an increased likelihood of gambling abstinence. In contrast, it may be seen as counterintuitive that advice about limit setting indeed was associated both with an increased likelihood of limit setting, but instead with a lower likelihood of gambling abstinence. However, it appears intuitive that a limit is chosen only by clients who choose to continue gambling to some extent, and that limit setting is unlikely in individuals who opt for a total gambling abstinence. For the same reason, information about limit setting is unlikely to be provided in a conversation with a client who actively states an intention or desire to stop gambling completely, and more likely to be provided to individuals who state an intention to gamble further. Also, it should be borne in mind that this part of the study cannot be considered to be controlled; it is likely that advice about setting limits was more likely to be delivered to people with particularly large needs for that, and where it is therefore less likely that gambling abstinence would occur. However, it can be assumed that for individuals who did set limits to their gambling postintervention, this should have had a reducing effect on their gambling practices. The effect of the telephone intervention on limit setting was consistent with the findings from Jonsson et al. [10], where limit setting was indeed more likely to occur in individuals who had been contacted by telephone, compared to the controls and compared to the postal letter condition. In contrast, no effect on selfexclusions were seen in that study [10], but it remains likely that a motivational telephone intervention indeed has an effect on active self-limiting practices in gambling.

This study assesses a short time frame after the intervention provided. Here, it should be borne in mind that the role of this type of brief intervention is to initiate change through a motivational process, rather than to shape a more long-term therapeutic process. Thus, a client reached with the present type of brief telephone intervention may be at a very early stage in a motivational change process, or before any perception of a need to change. However, it also cannot be excluded that in some clients, the intervention delivers a relieving first contact, which may represent a type of external intervention which the gambler has been sub-consciously waiting for, as part of an external control measure for an individuals in a situation of severe loss of internal control. Here, also, it should be borne in mind that being recruited to the study through a 'red' self-test was associated with increased likelihood of gambling abstinence, when controlling for the intervention vs control and for a number of other co-variates. One interpretation of this may that clients who take a self-test are already in a motivational process, and that this is an indicator of a more favorable course in the weeks following this measure. More research is needed in order to further 
highlight this, but the finding strengthens the rationale behind providing self-test for gambling problems to a gambling operator's clients.

Potential effects on subsequent treatment seeking go beyond the scope of the present study. Gambling disorder is a condition which can be treated [20]; different psycho-therapeutic approaches have been tested (and with hitherto less conclusive results also pharmacological strategies). Here, for example, cognitive-behavioral therapy is the treatment intervention most commonly applied. The relevance of a motivational telephone contact with high-risk gamblers can be seen in this context; a motivational conversation addressing a gambler's high-risk gambling practices may contribute to treatment seeking. It has been described that people's treatment seeking may be diverse [21], and that it may involve either formal seeking of professional specialized treatment, or the practice of initiating online support contacts of a less formal nature, or the initiation of contacts with self-help groups as available also in the present setting. In Sweden, self-help organizations are likely to be one of the more common providers of help and support for problem gamblers to contact [22]; a Swedish web survey demonstrated that around half of respondents from the general population would advise a person with gambling problems to seek that kind of support. In addition, social services and different health-care settings are available for the assessment and treatment of problem gambling [15]. Overall, based on the fact that treatment and support functions are increasingly available, this further underlines the need for motivational interventions which directly target gamblers in close association to their high-risk gambling behaviors.

Effects differed to some extent with respect to different gambling types. However, it was also clear that associations were substantially altered in the adjusted analyses, controlling for example for the extent of gambling and demographic data, compared to the occurrence of a favorable outcome in the unadjusted analyses. Slots casino gambling and live casino gambling were the most clearly associated with selfexclusion, while such an effect was not seen for the measures describing gambling abstinence. In the unadjusted analyses, obtaining gambling abstinence was more likely in online casino/bingo gamblers, where the intervention was significantly associated with abstinence, and less so in sports bettors, where abstinence for week 2-4 was associated with the intervention, whereas the overall abstinence measure was not. Thus, the study indicated a tendency towards a more extensive effect of the intervention in users of chance-based online gambling services. Online casino is more closely associated with addictive gambling in studies conducted recently in the present setting. This is particularly true when gambling practices of treatment-seeking gambling disorder patients are assessed [13], and in surveys in Swedish online gamblers, where online casino gambling is markedly more associated with indebtedness than online sports betting [23]. Thus, a possible interpretation is that online casino gambling may be associated with a particularly addictive behaviors and that an intervention, such as the one studied here, may hold promise there in particular.

There are few studies which can be compared to the present study results, as few studies applied a direct telephone intervention specifically $[6,10,11]$. Jonsson et al's study [11], however, demonstrated that in some gambling sub-types, a telephone intervention was clearly superior to a letter message to gamblers, and this included gamblers with extensive online casino or online sports betting, whereas a letter 
invitation may be sufficient in gamblers with high patterns of lottery gambling, i.e. intuitively in a gambling conditions with lower addictive potential. Thus, at least for the overall effect in online casino gamblers in the present study, this is in line with Jonsson et al's study [11]. Even up to one year postintervention, Jonsson and co-workers [10] also demonstrated an increased effect on active responsible gambling measures in gamblers receiving a telephone intervention compared to a control condition.

The present study controlled for the potential effects of COVID-19, as the sports lock-down following the onset of the pandemic is known to have changed gambling opportunities during the first few months [18]. Here, the month of April was considered to be the first month substantially affected in pre-T0 gambling practices, but also likely affected in the types of gambling available after TO. Here, differences in pre-T0 gambling were seen in April, consistent with what could be expected; less sports betting and a larger proportion of some non-sports gambling types. All four outcome measures assessed were more common in subjects included in April, but when conducting a sensitivity analyses excluding these clients, the overall outcome of the intervention was not changed. Thus, it could be concluded that the changes occurring to the gambling market during COVID-19 did not obviously affect the effectiveness of the motivational telephone intervention. Previous data have shown that in a different gambling operator, during the very first weeks of the pandemic in Sweden, there were modest alternations in the full extent of gambling practices [17]. Although the first weeks of the COVID-19 pandemic represents a unique and unforeseen change in the gambling markets, given the data above, it was not considered to disqualify the gamblers included in April, 2020, from the assessment of the method tested here.

\section{Limitations}

The present study is surrounded by certain limitations. A randomized controlled study design could not be applied here, and would require the gambling operator to randomize individuals in need for motivational support to either a motivational intervention or a non-intervention, and thereby without prior informed consent from the study subjects. Here, all individuals included were called, and subjects who were successfully reached were compared to their counterparts who - after three attempts - could not be reached. Thus, the randomization instead to a non-intervention would require either a prior consent procedure, which in itself may become a motivational intervention. However, given the intuitive need for support in individuals with highly hazardous gambling practices, it would be associated with ethical concerns if some individuals elected for the intervention would not be called.

The brief time frame of the follow-up procedure also may be seen as a limitation. However, it should be borne in mind that the intervention studied is brief in itself, and cannot be compared for example to a formal treatment intervention. Instead, it would be of great value to study whether individuals seek treatment after the intervention or whether they take action in their gambling on other operators. Such data cannot be obtained from the present study, and requires a more qualitative study component where gamblers themselves report events subsequent to the intervention call. Such study aims are included in a separate study [8]. 
In line with this, gambling data from other gambling operators could not be included here. It would be assumed in the study design that a motivational effect on gambling would also include a similar effect on the gambling on other operators, but such a hypothesis cannot be tested here. Also, it cannot be excluded that emotional reactions to the motivational call are negative, such that the effect on an individual's gambling on other operators would even be the opposite. Such a fear of negative reactions has been discussed, but hitherto has been shown to be limited [4]. Again, further study on the present motivational intervention has to be include other study designs, including subjective self-report data from gamblers, and even qualitative in-depth interviews assessing the users' experience from the intervention.

\section{Conclusions}

An active motivational telephone intervention, carried out by a state-owned gambling operator and addressing clients with a high-risk gambling practice, appeared to have promising effects, in comparison to control subjects who were included in the intervention but who could not be reached. Effects of a motivational telephone intervention may be more distinct with respect to active harm-reducing actions such as self-exclusion or gambling limit settings, whereas potential effects on gambling abstinence may be somewhat delayed, and may not be seen when assessing the full time period after the intervention. Users of online chance-based gambling types may have larger effects from a motivational telephone intervention than sports bettors. In the whole group of gamblers reached with a telephone intervention, active advisory content in the telephone call may increase the likelihood of self-exclusions or limit settings actually taking place. More in-depth studies of gambling after a motivational telephone intervention are needed, including assessments of user satisfaction and gambling outcomes in other gambling operators.

\section{Declarations}

\section{Ethics approval and consent to participate}

All data were derived from the client registers of AB Svenska Spel, and delivered to the first author in a fully de-identified format. The study was approved by the Swedish Ethics Authority (file number 2020/03281), and it was pre-registered on clinicaltrials.gov with the clinical trials identifier number NCT04646421 (registered 30/11/2020). All study participants were 18 years or older.

Based on the ethics application which has been approved by the Swedish Ethics Authority, the present study, involving retrospective data, did not require informed consent to be obtained from study participants. Thus, the ethics committee (Swedish Ethics Authority, etikprovning.se) is responsible of the decision that informed consent was not required in the study.

Study methods were fully performed in accordance with the ethics in research law in Sweden, and with the methodology approved by the Swedish Ethics Authority, and in accordance with the Declaration of Helsinki. 


\section{Consent for publication}

Not applicable. The manuscript does not contain data that can be identified to a specific individual.

\section{Availability of data and materials}

The datasets generated during and analyzed during the current study are not publicly available due to the fact that AB Svenska Spel owns the data, and because the ethics applications involved the full confidentiality and non-dissemination of data, such that dissemination of data would require updated requests to these bodies, but data are available from the corresponding author on reasonable request.

\section{Competing interests}

$\mathrm{AH}$ is a researcher employed by Lund University, and is not employed by AB Svenska Spel (the stateowned gambling operator operating under the license by the Swedish government). He has research funding from the research council of $A B$ Svenska Spel, and AB Svenska Spel supports his employment at Lund University financially. $\mathrm{AH}$ also has research funding from the research council of Systembolaget $A B$, which is the government alcohol monopoly of Sweden. KF, MD and AL are employed by AB Svenska Spel.

\section{Funding}

The work of $\mathrm{AH}$ in the present project is funded by his overall research funding and employment, which are supported by AB Svenska Spel and by the Region Skåne health care organization. All other authors are employed by AB Svenska Spel, which has also carried out data collection and technical support within its resources. No other external funding has been obtained for the study.

\section{Authors' contributions}

All authors were involved in the overall research idea and design. KF and MD have participated actively in the practical planning of the intervention itself. KF, MD and AL have been involved in the data collection procedures. All authors have actively participated in the interpretation of data and creation of the research discussion and conclusions. AH carried out the statistical analyses, and wrote the manuscript draft. All authors have been active in reviewing and editing the manuscript, and all authors have approved its final version and its submission.

\section{Acknowledgements}


The authors are grateful to employees of AB Svenska Spel involved in the data collection and technical support of the project, and to the employees involved in the work with motivational telephone interventions.

\section{References}

1. Potenza MN, Balodis IM, Derevensky J, Grant JE, Petry NM, Verdejo-Garcia A, Yip SW. Gambling disorder. Nat Rev Dis Prim. 2019;5:51.

2. Muggleton N, Parpart P, Newall P, Leake D, Gathergood J, Stewart N. The association between gambling and financial, social and health outcomes in big financial data. Nat Hum Behav. 2021;5:319-326.

3. Forsström D, Hesser H, Carlbring P. Usage of a responsible gambling tool: a descriptive analysis and latent class analysis of user behavior. J Gambl Stud. 2016;32:889-904.

4. Ivanova E, Rafi J, Lindner P, Carlbring P. Experiences of responsible gambling tools among nonproblem gamblers: a survey of active customers of an online gambling platform. Addict Behav Rep. 2019;9:100161.

5. Gainsbury SM, Angus DJ, Procter L, Blaszczynski A. Use of consumer protection tools on internet gambling sites: customer perceptions, motivators, and barriers to use. J Gambl Stud. 2020;36:25976.

6. Jonsson J, Hodgins DC, Munck I, Carlbring P. Reaching out to big losers: a randomized controlled trial of brief motivational contact providing gambling expenditure feedback. Psychol Addict Behav. 2019;33:179-189.

7. Forsström D, Cisneros Örnberg J. Responsible gambling in practice: a case study of views and practices of Swedish oriented gambling companies. Nordic Stud Alc Drugs 2019;36:91-107.

8. Håkansson A, Dahlström M, Franklin K, Lyckberg A. Responsible gambling telephone intervention to high-risk gamblers by a state-owned gambling operator in Sweden: study protocol for a study on effectiveness, user satisfaction, and acceptability. Int J Environ Res Publ Health 2020;17:9069.

9. Yakovenko I, Quigley L, Hemmelgarn BR, Hodgins DC, Ronksley P. The efficacy of motivational interviewing for disordered gambling: systematic review and meta-analysis. Addict Behav. 2015;43:72-82.

10. Jonsson J, Hodgins DC, Munck I, Carlbring P. Reaching out to big losers leads to sustained reductions in gambling over 1 year: a randomized controlled trial of brief motivational contact. Addiction 2020;115:1522-1531.

11. Jonsson J, Hodgins DC, Munck I, Carlbring P. (2021). Reaching out to big losers: how different types of gamblers are affected by a brief motivational contact initiated by the gambling provider. J Gambl Stud. 2021;37:387-401.

12. Abbott MW, Romild U, Volberg RA. The prevalence, incidence, and gender and age-specific incidence of problem gambling: results of the Swedish longitudinal gambling study (Swelogs). Addiction 
2018;113:699-707.

13. Håkansson $\mathrm{A}$, Mårdhed $\mathrm{E}$, Zaar $\mathrm{M}$. Who seeks treatment when medicine opens the door to gambling disorder patients - psychiatric co-morbidity and heavy predominance of online gambling. Front Psychiatry 2017;8:255.

14. Håkansson A, Widinghoff C. Television gambling advertisements: extent and content of gambling advertisements with a focus on potential high-risk commercial messages. Addict Behav Rep. 2019;9:100182.

15. Håkansson A, Ford M. The general population's view on where to seek treatment for gambling disorder - a general population survey. Psychol Res Behav Manag. 2019;12:1137-1146.

16. Jonsson J, Munck I, Volberg R, Carlbring P. (). GamTest: Psychometric Evaluation and the Role of Emotions in an Online Self-Test for Gambling Behavior. J Gambl Stud. 2017:1-19.

17. Lindner P, Forsström D, Jonsson J, Berman AH, Carlbring P. Transitioning between online gambling modalities and decrease in total gambling activity, but no indication of increase in problematic online gambling intensity during the first phase of the COVID-19 outbreak in Sweden: a time series forecast study. Front Publ Health 2020;8:554542.

18. Håkansson A. Brief research report: Impact of COVID-19 on online gambling - a general population survey during the pandemic. Front Psychol. 2020;11:568543.

19. IBM Corp. IBM SPSS Statistics for Windows, Version 25.0. Armonk, NY: IBM Corp, 2017.

20. Di Nicola M, De Creszenzo F, D’Alò GL, Remondi C, Panaccione I, Moccia L, et al. Pharmacological and psychosocial treatment of adults with gambling disorder: a meta-review. J Addict Med. 2020;14:e15-23.

21. Rodda SN, Dowling NA, Lubman DI. Gamblers seeking online help are active help-seekers: time to support autonomy and competence. Addict Behav. 2018;87:272-275.

22. Binde, P.A. Swedish mutual support society of problem gamblers. Int J Mental Health Addict, 2012;10:512-523.

23. Håkansson A, Widinghoff C. Over-indebtedness and problem gambling in a general population sample of online gamblers. Front Psychiatry 2020;11:7.

\section{Tables}

\section{Table 1}

Distribution over time of T0 date ( $\mathrm{N}=2,924)$, i.e. the date of either the motivational telephone call (intervention group) or the day of the last contact attempt for individuals who were not reached (control group). 


\begin{tabular}{|lll|}
\hline Month & $\mathbf{n}$ & \% \\
\hline September, 2019 & 241 & 8 \\
\hline October, 2019 & 384 & 13 \\
\hline November, 2019 & 334 & 11 \\
\hline December, 2019 & 396 & 14 \\
\hline January, 2020 & 398 & 14 \\
\hline February, 2020 & 367 & 13 \\
\hline March, 2020 & 413 & 14 \\
\hline April, 2020 & 391 & 13 \\
\hline & 2,924 & 100 \\
\hline
\end{tabular}

Table 2

Distribution of pre-T0 gambling practices in included individuals $(\mathrm{N}=2,924)$

\begin{tabular}{|lll|}
\hline Gambling types eight weeks prior to T0 & $\mathbf{n}$ & $\%$ \\
\hline Pool sports betting & 2,122 & 73 \\
\hline Other sports betting & 1,860 & 64 \\
Online poker & 616 & 21 \\
Online bingo & 208 & 7 \\
\hline Online casino slots & 1,262 & 43 \\
Online live casino & 750 & 26 \\
\hline Other online casino & 301 & 10 \\
\hline & & \\
\hline Any bingo/casino gambling, total & 1,651 & 56 \\
\hline Any sports betting, total & 2,360 & 81 \\
\hline & & \\
\hline
\end{tabular}

Table 3

Descriptive comparison of intervention and control groups $(\mathrm{N}=2,924)$. 


\begin{tabular}{|c|c|c|c|c|}
\hline & $\begin{array}{l}\text { Intervention } \\
(\mathrm{n}=1,420), \% \text { (n) }\end{array}$ & $\begin{array}{l}\text { Control }(n=1,504), \\
\%(n)\end{array}$ & $p$ value & $\begin{array}{l}\text { Missing } \\
\text { data, } \\
\text { total }\end{array}$ \\
\hline Female gender & $6(86)$ & $10(155)$ & $<0.001$ & 1 \\
\hline $\begin{array}{l}\text { Mean age, mean } \\
\text { (SD) }\end{array}$ & 39.3 (13.9) & $37.4(13.8)$ & $<0.001$ & 9 \\
\hline $\begin{array}{l}\text { Gambling } \\
\text { frequency, } \\
\text { days/week, mean } \\
\text { (SD) }\end{array}$ & $3.3(2.1)$ & $3.3(2.0)$ & 0.80 & 0 \\
\hline $\begin{array}{l}\text { Losses } 28 \text { days, } \\
\text { mean (SD) }\end{array}$ & $23,585(31,132)$ & $20,485(22,879)$ & $<0.01$ & 138 \\
\hline Self-test present & $17(235)$ & $18(271)$ & 0.29 & 0 \\
\hline $\begin{array}{l}\text { Gambling types, } \\
\text { past } 8 \text { weeks }\end{array}$ & & & & 0 \\
\hline $\begin{array}{l}\text { - pool sports } \\
\text { betting }\end{array}$ & $73(1,037)$ & $72(1,085)$ & 0.59 & \\
\hline $\begin{array}{l}\text { - other sports } \\
\text { betting }\end{array}$ & $62(885)$ & 65 (975) & 0.16 & \\
\hline - online poker & $21(303)$ & $21(313)$ & 0.73 & \\
\hline - online bingo & $6(92)$ & $8(116)$ & 0.19 & \\
\hline $\begin{array}{l}\text { - } \quad \text { slots casino } \\
\text { gambling }\end{array}$ & $41(582)$ & $45(680)$ & 0.02 & \\
\hline - live casino & $26(367)$ & $25(383)$ & 0.81 & \\
\hline - other casino & $10(144)$ & $10(157)$ & 0.79 & \\
\hline
\end{tabular}

Table 4

Gambling abstinence outcome data in sub-groups of included participants. 


\begin{tabular}{|c|c|c|c|c|c|c|}
\hline & $\begin{array}{l}\text { Abstinence, } \\
\text { intervention, } \\
\% \text { (n) }\end{array}$ & $\begin{array}{l}\text { Abstinence, } \\
\text { control, \% } \\
\text { (n) }\end{array}$ & $p$ value & $\begin{array}{l}\text { Week 2-4 } \\
\text { abstinence, } \\
\text { intervention, } \\
\%(n)\end{array}$ & $\begin{array}{l}\text { Week 2-4 } \\
\text { abstinence, } \\
\text { control, \% } \\
\text { (n) }\end{array}$ & $p$ value \\
\hline All & $18(251)$ & 15 (229) & 0.07 & 24 (334) & $20(301)$ & 0.02 \\
\hline$<10,000$ & $20(96)$ & 20 (117) & 0.89 & 27 (131) & $26(153)$ & 0.56 \\
\hline $10,000-19,999$ & $16(40)$ & $10(27)$ & 0.06 & $21(53)$ & $14(38)$ & $0.05^{\star}$ \\
\hline $20,000-29,999$ & $12(22)$ & $11(20)$ & 0.77 & $19(34)$ & $13(23)$ & 0.12 \\
\hline $30,000-39,999$ & $12(21)$ & $14(22)$ & 0.54 & $15(27)$ & $18(28)$ & 0.51 \\
\hline$>40,000$ & $23(46)$ & $13(24)$ & 0.01 & $28(58)$ & $19(35)$ & 0.02 \\
\hline Self-test & $21(50)$ & $27(72)$ & 0.17 & $28(65)$ & 33 (89) & 0.21 \\
\hline No self-test & $17(201)$ & $13(157)$ & $<0.01$ & $23(269)$ & $17(212)$ & $<0.001$ \\
\hline Women & $21(18)$ & $17(26)$ & 0.42 & $26(22)$ & $25(40)$ & 0.94 \\
\hline Men & $17(233)$ & $15(203)$ & 0.09 & 23 (312) & $19(262)$ & 0.01 \\
\hline $\begin{array}{l}\text { Any } \\
\text { pool/sports } \\
\text { betting pre-T0 }\end{array}$ & $13(148)$ & 12 (149) & 0.56 & 19 (211) & $16(194)$ & 0.09 \\
\hline $\begin{array}{l}\text { Any } \\
\text { casino/bingo } \\
\text { gambling pre- } \\
\text { T0 }\end{array}$ & 19 (148) & $14(124)$ & $<0.01$ & 24 (186) & $20(171)$ & 0.03 \\
\hline $\begin{array}{l}\text { Any poker } \\
\text { gambling pre- } \\
\text { T0 }\end{array}$ & $16(48)$ & $11(35)$ & 0.09 & $20(61)$ & $16(49)$ & 0.15 \\
\hline Age 18-24 & $23(59)$ & $22(77)$ & 0.72 & 31 (79) & $28(98)$ & 0.41 \\
\hline Age 25-39 & $19(93)$ & $18(94)$ & 0.68 & 24 (119) & $24(126)$ & 0.94 \\
\hline Age 40-49 & $17(50)$ & $9(26)$ & $<0.01$ & $21(63)$ & $13(38)$ & $<0.01$ \\
\hline Age 50- & $13(47)$ & $10(32)$ & 0.21 & $20(70)$ & $12(39)$ & $<0.01$ \\
\hline
\end{tabular}

*above 0.05 , rounded off to two decimals 
Logistic regression, variables in association with gambling abstinence post-T0 (including cases with available data on all variables, $n=2,924$ )

\begin{tabular}{|llll|}
\hline & OR & 95 percent confidence interval & $\boldsymbol{p}$ \\
\hline Age & 0.98 & $0.97-0.99$ & $<0.001$ \\
\hline Female gender & 0.80 & $0.55-1.18$ & 0.27 \\
\hline Pool sports games & 0.41 & $0.32-0.52$ & $<0.001$ \\
\hline Sports betting & 0.44 & $0.34-0.56$ & $<0.001$ \\
\hline Online poker & 0.52 & $0.39-0.71$ & $<0.001$ \\
\hline Online casino & 0.96 & $0.65-1.40$ & 0.82 \\
\hline Online bingo & 0.63 & $0.38-1.05$ & 0.07 \\
\hline Slots gambling & 0.69 & $0.55-0.87$ & $<0.01$ \\
\hline Live casino gambling & 1.17 & $0.90-1.52$ & 0.25 \\
\hline Intervention vs control & 1.22 & $0.98-1.52$ & 0.08 \\
\hline Self-test & 1.81 & $1.33-2.44$ & $<0.001$ \\
\hline Past-28-day losses & 1.00 & $0.98-1.02$ & 0.94 \\
\hline & & & \\
\hline
\end{tabular}

\section{Table 6}

Logistic regression, variables in association with non-gambling week 2-4 post-T0 (including cases with available data on all variables, $n=2,679$ ) 


\begin{tabular}{|llll|}
\hline & OR & 95 percent confidence interval & $\boldsymbol{p}$ \\
\hline Age & 0.98 & $0.97-0.99$ & $<0.001$ \\
\hline Female gender & 0.86 & $0.61-1.22$ & 0.40 \\
\hline Pool sports games & 0.39 & $0.31-0.48$ & $<0.001$ \\
\hline Sports betting & 0.45 & $0.36-0.56$ & $<0.001$ \\
\hline Online poker & 0.51 & $0.39-0.67$ & $<0.001$ \\
\hline Online casino & 1.22 & $0.87-1.71$ & 0.24 \\
\hline Online bingo & 0.89 & $0.59-1.33$ & 0.57 \\
\hline Slots gambling & 0.65 & $0.53-0.81$ & $<0.001$ \\
\hline Live casino gambling & 1.10 & $0.86-1.40$ & 0.44 \\
\hline Intervention vs control & 1.28 & $1.05-1.56$ & 0.01 \\
\hline Self-test & 1.59 & $1.20-2.10$ & $<0.01$ \\
\hline Past-28-day losses & 1.00 & $0.98-1.01$ & 0.71 \\
\hline
\end{tabular}

Table 7

Logistic regression, variables in association with self-exclusion post-T0 (including cases with available data on all variables, $n=2,679$ ) 


\begin{tabular}{|llll|}
\hline & OR & 95 percent confidence interval & $\boldsymbol{p}$ \\
\hline Age & 1.02 & $1.01-1.03$ & $<0.001$ \\
\hline Female gender & 1.15 & $0.72-1.82$ & 0.56 \\
\hline Pool sports games & 0.87 & $0.62-1.22$ & 0.41 \\
\hline Sports betting & 1.19 & $0.86-1.65$ & 0.28 \\
\hline Online poker & 0.99 & $0.70-1.39$ & 0.93 \\
\hline Online casino & 0.85 & $0.55-1.30$ & 0.45 \\
\hline Online bingo & 0.50 & $0.90-2.31$ & 0.06 \\
\hline Slots gambling & 3.18 & $2.36-4.29$ & $<0.001$ \\
\hline Live casino gambling & 1.72 & $1.26-2.35$ & $<0.001$ \\
\hline Intervention vs control & 1.55 & $1.18-2.04$ & $<0.01$ \\
\hline Self-test & 0.64 & $0.39-1.03$ & 0.07 \\
\hline Past-28-day losses & 1.01 & $0.99-1.03$ & 0.37 \\
\hline
\end{tabular}

Table 8

Logistic regression, variables in association with limit setting post-T0 (including cases with available data on all variables, $n=2,679$ ) 


\begin{tabular}{|llll|}
\hline & OR & 95 percent confidence interval & $\boldsymbol{p}$ \\
\hline Age & 1.00 & $0.99-1.01$ & 0.81 \\
\hline Female gender & 1.32 & $0.78-2.23$ & 0.29 \\
\hline Pool sports games & 0.90 & $0.62-0.30$ & $<0.001$ \\
\hline Sports betting & 1.21 & $0.85-1.73$ & 0.29 \\
\hline Online poker & 0.81 & $0.55-1.19$ & 0.29 \\
\hline Online casino & 1.64 & $1.03-2.62$ & 0.04 \\
\hline Online bingo & 1.11 & $0.63-1.95$ & 0.73 \\
\hline Slots gambling & 1.18 & $0.86-1.62$ & 0.31 \\
\hline Live casino gambling & 0.82 & $0.56-1.19$ & 0.29 \\
\hline Intervention vs control & 2.28 & $1.67-3.12$ & $<0.001$ \\
\hline Self-test & 0.69 & $0.41-1.16$ & 0.16 \\
\hline Past-28-day losses & 1.02 & $1.00 *-1.04$ & 0.08 \\
\hline *below 1.00, rounded off to two decimals & \\
\hline
\end{tabular}

Figures 
Introduction of the caller and the reason for the call, and asking for permission to continue the conversation.

Question about the client's believed level of losses, and (if accepted by the client) information about her/his losses, in an attempt to create a motivational process. Information about level of losses per month. Further conversation depending on the client's reaction to this.

Attention to the client's reaction and to motivational, change-oriented content in the client's reaction.

Open-ended questions, reflective listening. Positive feed-back to change talk detected in the client's reaction.

In case of willingness to change, active help with either setting or lowering of gambling limits, blocking increase of limits, self-exclusion, or advice about official national selfexclusion program.

Assistance in creating an action plan for the client's future gambling behavior.

Summary of what has been said and agreed during the conversation.

\section{Figure 1}

Overall agenda in motivational calls. 